\title{
Calidad de las estadísticas de mortalidad materna en Cuba, 2013
}

\author{
Sonia Bess Constantén, ${ }^{1}$ Miguel Ángel Martínez Morales, ${ }^{1}$ María Rosa \\ Fernández Viera, ${ }^{1}$ Vanessa Mazorra Ramos, ${ }^{1}$ Ismell Alonso Alomá, ${ }^{1}$ Libia \\ Margarita López Nistal, ${ }^{1}$ Miriam Alicia Gran Álvarez, ${ }^{1}$ Roberto Álvarez Fumero ${ }^{1}$ \\ y Mercedes Piloto Padrón ${ }^{1}$
}

Forma de citar

Bess Constantén S, Martínez Morales MA, Fernández Viera MR, Mazorra Ramos V, Alonso Alomá I, López Nistal LM, et al. Calidad de las estadísticas de mortalidad materna en Cuba, 2013. Rev Panam Salud Publica. 2018;42:e47. doi: 10.26633/RPSP.2018.47.

RESUMEN Objetivos. Evaluar el grado de integridad del registro estadístico cubano de muertes maternas y la calidad de la clasificación de las causas de muerte recogidas en ese registro.

Métodos. Se analizó la información de todas las cubanas fallecidas en edad fértil en el año 2013 según el registro continuo de mortalidad de la Dirección de Registros Médicos y Estadísticas de Salud del Ministerio de Salud Pública de Cuba (MINSAP), independientemente de la causa básica de muerte consignada. Cuatro grupos de investigación (nacional, de expertos, provincial y de unidades de salud), con funciones definidas, aplicaron cuatro formularios para determinar si hubo embarazo en el año previo a la defunción, reevaluar si los casos correspondian a una muerte materna y, en ese caso, revisar la clasificación.

Resultados. Se investigaron las 2731 mujeres fallecidas en edad reproductiva notificadas en Cuba en el 2013; de ellas las causas de muerte de 2711 (99,3\%) resultaron concluyentes y, de estas, 97 (3,6\%) habian tenido un embarazo en el año previo a la defunción. Se encontraron 50 muertes maternas (una más que en el registro continuo) para $2 \%$ de error y un factor de ajuste de 1,02. De las 97 muertes estudiadas, solo se reclasificaron 4 casos: 2 muertes maternas y 1 muerte relacionada con el embarazo, el parto y el puerperio, según el registro continuo, que se reclasificaron como muertes directas, y 1 muerte considerada no materna por el registro continuo que se reclasificó como muerte materna tardía, para una concordancia de 95,9\%.

Conclusiones. La información sobre las muertes maternas recogida en el registro continuo de mortalidad del MINSAP tiene un alto nivel de integridad. La calidad de la clasificación de las muertes maternas en ese registro es elevada, al existir muy pocos cambios en la reclasificación de las causas de muerte.

Palabras clave Mortalidad materna; vigilancia epidemiológica; control de calidad; certificado de defunción; Cuba.

La muerte de una mujer durante el embarazo, el parto o el puerperio está considerada como una manifestación de fracaso en la atención a la salud sexual y

\footnotetext{
Ministerio de Salud Pública, La Habana, Cuba. Enviar la correspondencia a Miguel Ángel

Martínez Morales, mangel@mspdne.sld.cu
}

reproductiva. Su desconocimiento y altos índices en muchos países de escasos recursos son un testimonio del descuido de esta prioridad de salud (1-5).

La razón de mortalidad materna es un indicador fiel de la inequidad, la desigualdad socioeconómica de la mujer y de su deficiente acceso a los servicios de salud (1, 6-9). Aunque la reducción de este parámetro ha sido uno de los principales objetivos de conferencias internacionales $(2,10)$, su medición es compleja, por lo que resulta difícil comparar los datos obtenidos por diferentes fuentes y

Este es un articulo de acceso abierto distribuido bajo los terminos de la licencia Creative Commons Attribution-NonCommercial-NoDerivs 3.0 IGO, que permite su uso, distribución y reproducción en cualquier medio, siempre que el trabajo original se cite de la manera adecuada. No se permiten modificaciones a los artículos ni su uso comercial. Al reproducir un artículo no debe haber ningún indicio de que la OPS o el artículo avalan a una organización o un producto específico. El uso del logo de la OPS no está permitido. Esta leyenda debe conservarse, junto con la URL original del artículo. 
todavía se debaten aspectos metodológicos relacionados con su definición y la selección de las variables que influyen en su comportamiento (10-13).

Una de las causas del desconocimiento del nivel real de mortalidad materna es la subestimación por subregistro, que varía según los países: en los llamados países desarrollados el subregistro de causas o las deficiencias de clasificación ocurren cuando la causa registrada del deceso no revela el verdadero vínculo entre el embarazo y la muerte materna; mientras, en los países en desarrollo incide también la ausencia de sistemas de medición eficientes, lo que obstaculiza la notificación y limita las posibilidades de realizar comparaciones internacionales (11-15).

La Organización Mundial de la Salud, el Fondo de las Naciones Unidas para la Infancia (UNICEF), el Fondo de Población de las Naciones Unidas y el Banco Mundial, en alianza con sectores académicos y a través del Grupo Interagencial para la Estimación de la Mortalidad Materna (MMEIG, por sus siglas en inglés), han desarrollado modelos estadísticos de estimación del indicador a partir de la aplicación de métodos de ajuste $(16,17)$. En el 2013, el MMEIG ubicó a Cuba entre los países con buena información, pero sin investigaciones documentadas sobre las defunciones de mujeres en edad fértil. Por esto, corrigieron los datos de origen por un factor de ajuste de 1,5-por ser la mediana de los resultados de estudios realizados por otros países (17, 18) — que aún se aplica en la actualidad. Sin embargo, en el caso de Cuba esto incrementa el valor de ese indicador más de lo razonable.

Analizar las muertes maternas permite identificar las causas de muerte, proporciona evidencia de la calidad del monitoreo y facilita la evaluación del cumplimiento de los programas dirigidos a la atención de la mujer. La integridad de los registros y la clasificación adecuada de las causas de muertes son elementos muy importantes y su estudio debe adaptarse a las condiciones concretas de cada país (19).

Las características del Sistema Nacional de Salud de Cuba - como sistema único y de cobertura universal y gratuita (20) - favorecen que la información se capte en todos los niveles del sistema por personal capacitado y que esta fluya por la estructura estadística de salud mediante un registro mixto, que combina el procesamiento manual y el automatizado.

Los certificados de defunción transitan paralelamente por el sector de la salud, la Oficina Nacional de Estadísticas y el Registro del Estado Civil, y periódicamente se comprueba la compatibilidad de estos datos. Los enterramientos solo se realizan en cementerios públicos y para ello es requisito indispensable presentar el certificado de defunción, confeccionado y firmado siempre por un médico $(21,22)$.

Con frecuencia, en el país se ejecutan estudios sobre la calidad de las estadísticas de mortalidad $(23,24)$. En el caso de la mortalidad materna se emplea un registro continuo a cargo del Ministerio de Salud Pública de Cuba (MINSAP), con cobertura nacional, cuya calidad se garantiza mediante verificaciones y auditorías periódicas (25); esta información se considera fiable. También se utilizan sistemas de medición paralelos y fuentes independientes de comparación intencionales, para la búsqueda activa y permanente de errores en los procesos de registro continuo para lograr una mayor completitud y veracidad (26). No obstante, como todas las fuentes de datos, tiene sus limitaciones para estimar los verdaderos índices de mortalidad materna $(7,10,11,13)$.

En el 2013, el MMEIG exhortó a Cuba a emprender estudios sobre las defunciones de mujeres en edad fértil para actualizar el conocimiento, adoptar mejores decisiones y mejorar el proceso de estimación (17). El Estudio de Mortalidad en Mujeres en Edad Reproductiva (27) y el Estudio de Búsqueda Intencionada y Reclasificación de Muertes Maternas (19) - conocidos respectivamente como RAMOS y BIRMM, por sus siglas en inglés- permiten investigar las causas de muerte en mujeres fallecidas en edad reproductiva y se aplican tanto en países con buenos sistemas de registros vitales para identificar errores de clasificación como en países sin esos registros.

El objetivo de este trabajo es evaluar el grado de integridad del registro estadístico cubano de muertes maternas y la calidad de la clasificación de las causas de muerte recogidas en ese registro.

\section{MATERIALES Y MÉTODOS}

Se realizó un estudio descriptivo de corte transversal, según la metodología utilizada en RAMOS (27), a partir de los datos de todas las mujeres fallecidas en Cuba en el 2013 en edad fértil (entre 12 y 49 años), asentados en la base de datos del registro continuo de mortalidad de la Dirección de Registros Médicos y Estadísticas de Salud del MINSAP, independientemente de la causa básica de muerte consignada. En los casos de fallecimiento en estas edades, está establecido que en el Certificado Médico de Defunción se indique si la fallecida tuvo un embarazo en el año previo a su defunción.

La integridad se calculó como la razón del número de muertes maternas identificadas por la investigación entre las notificadas en el registro; se calculó así para poder comparar los resultados con las referencias del MMEIG. El valor ideal es la unidad. Según los criterios del grupo de expertos, se aceptó un error de hasta $6 \%$ para considerar un reporte como íntegro.

La calidad del registro se calculó como el porcentaje de muertes maternas clasificadas en esta investigación de manera diferente a la consignada en el registro. Se consideró como calidad adecuada una discordancia inferior a $6 \%$.

\section{Estrategia de investigación}

Para el estudio se crearon cuatro grupos de investigadores, conformados por especialistas en estadística (bioestadísticos, licenciados en gestión de la información y estadísticos) y de los servicios de atención primaria y hospitalaria relacionados con la atención maternoinfantil (ginecoobstetras, médicos, personal de enfermería y patólogos); cada grupo tenía funciones específicas:

- Grupo nacional: Condujo el estudio.

- Grupo de expertos: Seleccionado por el grupo nacional - por su calificación, nivel de actualización en el tema, no vinculación con la recogida y la codificación de los datos de mortalidad, y capacidad de arbitraje para la certificación y la codificación de las causas de muerte-, este grupo asesoró el proceso y reevaluó todos los casos con sospecha de muerte materna, así como las defunciones cuyas causas no resultaron concluyentes.

- Grupo provincial: Controló la distribución y la digitalización de la información y evaluó su calidad; además, envió los formularios de las fallecidas en su territorio hacia sus provincias de residencia cuando eran foráneas. 
- Unidades de salud (hospitales y áreas comunitarias de salud): Llenaron los formularios y los enviaron al grupo provincial.

Se compararon los resultados obtenidos con lo reflejado en el registro continuo del MINSAP, según tres variables:

1. Territorio de residencia de la fallecida: Se asignó alguna de las 15 provincias o el municipio especial Isla de la Juventud, según la dirección que apareciera en el carné de identidad de la fallecida.

2. Muerte materna: Si la muerte se clasificó como materna o no. Cada caso se codificó según la Clasificación Estadística Internacional de Enfermedades y Problemas Relacionados con la Salud en su 10. ${ }^{a}$ revisión (CIE-10) (28) como:

a. muerte materna, en las que se incluyen las muertes directas y las indirectas

b. otras muertes relacionadas con el embarazo, el parto y el puerperio

c. muertes maternas tardías

d. no clasifica como muerte materna.

3. Embarazo previo: Indica el resultado de la aplicación de los formularios 1-4 para comprobar si la fallecida tuvo un embarazo en el año previo a su defunción. El resultado pudo ser:

a. Sí, si se comprobó que tuvo un embarazo el año anterior.

b. No, si se comprobó que no tuvo un embarazo el año anterior.

c. No se sabe, cuando no se pudo comprobar la existencia o no de un embarazo el año anterior.

\section{Procedimiento}

Se identificó en la base de datos a todas las fallecidas de 12 a 49 años de edad. El grupo nacional revisó los certificados médicos de defunción de todas las fallecidas en estudio y tres miembros de los equipos de investigación de los diferentes niveles clasificaron las muertes según el sitio de la defunción en hospitalarias o extrahospitalarias, y aplicaron los formularios correspondientes (figura 1). En todos los casos se exigió como documentación adicional la historia clínica, el protocolo de necropsia y la discusión de fallecido.

- Formulario 1: A cargo de los médicos de asistencia. Se aplicó en los casos de fallecidas hospitalizadas. Cuando se

\section{FIGURA 1. Diagrama de la aplicación de los formularios}

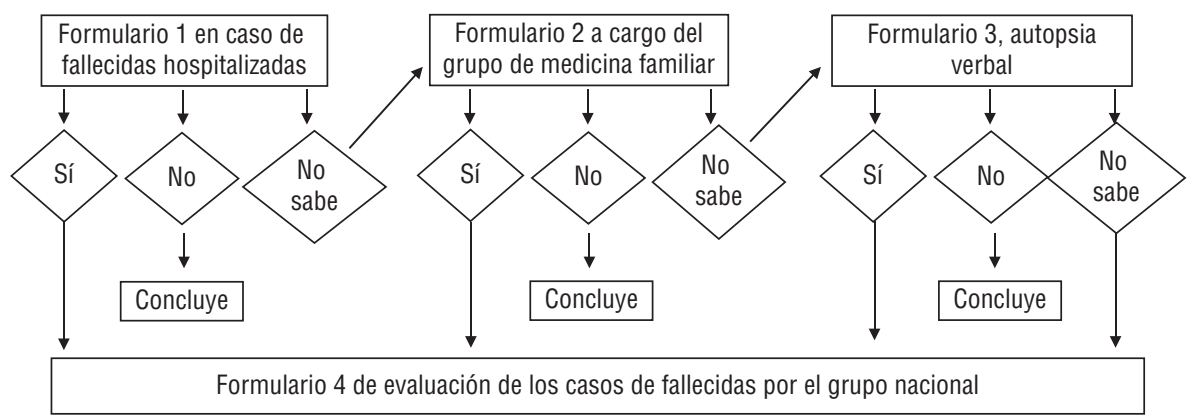

Nota: El resultado esperado de los formularios 1, 2 y 3 es la existencia de un embarazo en el año previo a la defunción. Fuente: Elaboración de los autores.

pudo concluir que hubo o no un embarazo el año previo al fallecimiento, los formularios se enviaron con la documentación adicional de rigor al grupo nacional. Si no se pudo comprobar, se aplicó el formulario 2. La fuente de información fueron las historias clínicas.

- Formulario 2: A cargo del grupo básico de salud comunitaria. Se aplicó en los casos de fallecidas no hospitalizadas y cuando en el formulario 1 no se logró definir si hubo o no un embarazo el año previo al fallecimiento. Cuando se logró una respuesta categórica al respecto, los formularios se enviaron al grupo nacional con la documentación adicional; de lo contrario, se aplicó el formulario 3. Las fuentes de información fueron los integrantes del equipo de medicina familiar que atendieron a la mujer en su área de atención de salud o residencia y los documentos de servicios prestados existentes en los consultorios del Programa del Médico y la Enfermera de la Familia, fundamentalmente, la historia clínica individual y la historia clínica familiar.

- Formulario 3 (autopsia verbal): A cargo del equipo básico de salud comunitaria. Se empleó en los casos en que en el formulario 2 no se logró definir si hubo o no un embarazo el año previo al fallecimiento. Este formulario se aplicó a un conviviente en el hogar de la fallecida, preferiblemente - y en este orden - al esposo o pareja de la mujer, la madre o el padre, los hermanos, o los hijos mayores de 18 años. Si ninguna de estas personas estaba disponible, se entrevistó a un vecino cercano a la familia que conociera los aspectos de interés de la mujer en el año previo a su defunción. En cualquiera de los casos, este formulario se envió con la documentación adicional al grupo nacional.

- Formulario 4: A cargo del grupo de expertos. El grupo de expertos elaboró 117 formularios y a partir de ellos se estableció la calidad de la clasificación de las 97 muertes maternas de las fallecidas que tuvieron un embarazo el año previo a su defunción, según los diferentes formularios, y los 20 casos en los que no se logró establecer ese elemento.

Los formularios - validados por el grupo nacional de investigación y el grupo de expertos con los datos de una muestra de fallecidas de la provincia La Habana- contenían preguntas cerradas (de respuesta única y excluyente) y abiertas.

El grupo nacional rehízo y recodificó las causas de muerte expuestas en cada certificado de defunción. Finalmente se clasificaron y compararon con los resultados del sistema de información del registro continuo del MINSAP.

Todos los datos obtenidos se digitalizaron en bases de datos confeccionadas en Microsoft Excel 2007 en los departamentos provinciales de Registros Médicos y Estadísticas de Salud correspondientes y se enviaron junto con el formato impreso y la información adicional recogida a la Dirección de Registros Médicos y Estadísticas de Salud del MINSAP.

La información se procesó mediante el programa estadístico SPSS versión 11.5 y se resumió en valores agregados, e indicadores porcentuales y de razón, con apertura territorial.

Para garantizar el cumplimiento de los requisitos éticos de la investigación, 
a todos los investigadores se les explicó el motivo del estudio, la metodología, las funciones, los resultados esperados, la confidencialidad, el tratamiento ético de la información, la necesidad de garantizar el anonimato de las fallecidas en los resultados, así como el compromiso y la aceptación de participar como investigador y permanecer como tal durante todo el estudio. Antes de entrevistar a familiares, convivientes y vecinos, se les solicitó su consentimiento para responder las preguntas necesarias. El protocolo de investigación fue aprobado por las autoridades del MINSAP según los requisitos del comité de ética de ese organismo.

\section{RESULTADOS}

\section{Formularios aplicados}

De las 2731 fallecidas investigadas, 1389 (50,9\%) murieron hospitalizadas y, de este grupo, en 1127 (81,1\%) se logró definir la existencia o no de un embarazo el año previo a su defunción y se comprobó este hecho en 63 (5,6\%) fallecidas. Se procedió a aplicar el formulario 2 a los $262(18,9 \%)$ casos en los que no se pudo establecer si hubo un embarazo el año anterior.

Se aplicaron 1604 formularios 2 a los 1342 casos de muertes extrahospitalarias y los 262 casos de muertes hospitalarias en los que no se pudo comprobar con el formulario 1 si hubo o no un embarazo previo. De ese total, en 1365 (85,1\%) se logró establecer definitivamente si hubo o no un embarazo el año anterior a su

FIGURA 2. Búsqueda intencionada y reclasificación de muertes maternas. Cuba, 2013

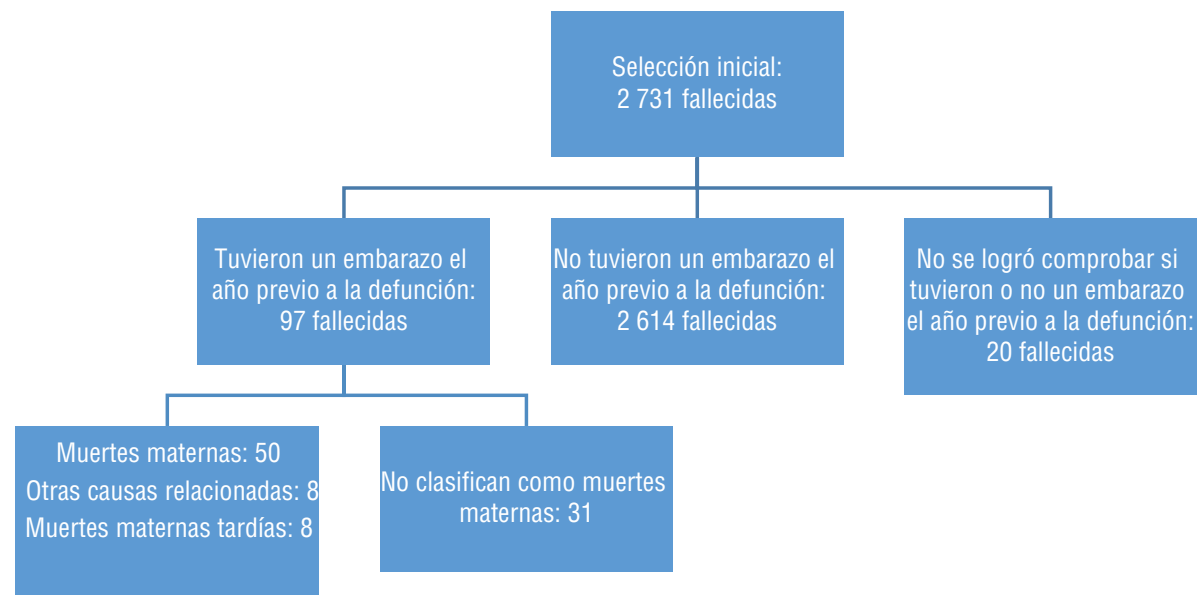

Fuente: Elaboración de los autores a partir de los resultados presentados. defunción; 33 (2,4\%) de ellas tuvieron un embarazo el año previo.

Según estaba previsto en la metodología, se aplicó el formulario 3 a los 239 $(14,9 \%)$ casos en los que no se pudo comprobar si hubo o no un embarazo el año previo al fallecimiento. Se logró verificar este elemento en 219 (91,6\%) casos, de los cuales 1 caso $(0,5 \%)$ había tenido efectivamente un embarazo el año anterior (figura 2).

Seguidamente se aplicó el formulario 4 a 117 casos: 97 fallecidas en las que en los formularios 1, 2 y 3 se comprobó que habían tenido un embarazo el año previo al fallecimiento y 20 fallecidas en las que la autopsia verbal no dio un resultado definitivo al respecto.

\section{Integridad del registro continuo de muertes maternas}

De las 2731 (100\%) mujeres fallecidas en edad reproductiva notificadas en el 2013 en Cuba, en 2711 (99,3\%) se pudo comprobar si tuvieron o no un embarazo el año previo a su defunción y 117 (4,2\%) tuvieron efectivamente un embarazo en el año anterior (figura 2).

Como resultado de la aplicación del formulario 4 a esos 117 casos, se determinó que 50 correspondieron a muertes maternas; de ellas, 30 directas y 20 indirectas. Además, se clasificaron 8 como otras muertes relacionadas con el embarazo y 8 como muertes maternas tardías (figura 2).

El grupo nacional determinó que 31 fallecidas de las 97 que habían tenido un embarazo el año previo a su

2731 fallecidas previo a la defunción. 2614 fallecidas tuvieron o no un embarazo l año previo a la defunción 20 fallecidas defunción no eran muertes maternas y otras 20 permanecieron con la clasificación de causas de muerte no maternas, al no hallarse evidencias para otro juicio tras la aplicación de los tres formularios y el resto de los procedimientos de pesquisa del estudio.

El estudio halló solo una muerte materna más (clasificada como directa) que las notificadas por el registro continuo del MINSAP ( $2 \%$ de error), por lo que el registro cubano de muertes maternas se puede considerar íntegro, según la categoría preconsensuada en las variables de la investigación. El factor de ajuste para Cuba en el 2013, resultante de este estudio, fue de 1,02 (cuadro 1 ).

\section{Calidad del registro continuo de muertes maternas}

Se confirmaron los 27 casos clasificados como muertes maternas directas en el registro continuo del MINSAP en el 2013 y los 7 casos clasificados como muertes maternas tardías (cuadro 2).

En el presente estudio se identificaron:

- Dos muertes maternas indirectas y una clasificada como "otras" (relacionadas con el embarazo, el parto y el puerperio), según el registro continuo del MINSAP, que se reclasificaron como muertes maternas directas en este estudio.

- Una muerte materna tardía más, que no estaba considerada como muerte materna en el registro.

Las variaciones se observaron en La Habana y Camagüey —una muerte materna indirecta se reclasificó como muerte materna directa en cada una de esas provincias-, Santiago de Cuba —una muerte clasificada como "otras" pasó a muerte materna directa-y Guantánamo, donde se registró la muerte materna tardía nueva (cuadro 1).

En consecuencia, la concordancia de la clasificación de las muertes maternas entre el registro continuo del MINSAP y este estudio fue de $95,9 \%$, por lo que se puede considerar que la calidad del registro continuo de muertes maternas es buena.

Lo anterior se confirmó al analizar las causas de la mortalidad materna directa según la causa básica de muerte notificada por el registro continuo del MINSAP y por este estudio. El orden de las causas no cambió y el número de fallecidas varió solo en una unidad en tres de las seis 
CUADRO 1. Muertes maternas según el tipo, los territorios y el método de registro. Cuba, 2013

\begin{tabular}{|c|c|c|c|c|c|c|c|c|c|c|}
\hline \multirow{2}{*}{ Territorios } & \multicolumn{2}{|c|}{ MM directas } & \multicolumn{2}{|c|}{ MM indirectas } & \multicolumn{2}{|c|}{ Otras MM } & \multicolumn{2}{|c|}{ MM tardías } & \multicolumn{2}{|c|}{ Total } \\
\hline & $\mathrm{RC}$ & Este estudio & $\mathrm{RC}$ & Este estudio & $\mathrm{RC}$ & Este estudio & $\mathrm{RC}$ & Este estudio & $\mathrm{RC}$ & Este estudio \\
\hline Pinar del Río & 0 & 0 & 0 & 0 & 0 & 0 & 0 & 0 & 0 & 0 \\
\hline Artemisa & 1 & 1 & 0 & 0 & 0 & 0 & 0 & 0 & 1 & 1 \\
\hline Mayabeque & 2 & 2 & 1 & 1 & 0 & 0 & 0 & 0 & 3 & 3 \\
\hline Matanzas & 2 & 2 & 0 & 0 & 0 & 0 & 0 & 0 & 2 & 2 \\
\hline Sancti Spíritus & 2 & 2 & 1 & 1 & 0 & 0 & 0 & 0 & 3 & 3 \\
\hline Ciego de Ávila & 2 & 2 & 1 & 1 & 0 & 0 & 1 & 1 & 4 & 4 \\
\hline Camagüey & 2 & 3 & 4 & 3 & 0 & 0 & 1 & 1 & 7 & 7 \\
\hline Las Tunas & 2 & 2 & 0 & 0 & 0 & 0 & 0 & 0 & 2 & 2 \\
\hline Holguín & 1 & 1 & 1 & 1 & 1 & 1 & 1 & 1 & 4 & 4 \\
\hline Total & 27 & 30 & 22 & 20 & 9 & 8 & 7 & 8 & 65 & 66 \\
\hline
\end{tabular}

Nota: MM: muertes maternas; RC: registro continuo.

Fuente: Elaboración de los autores a partir de los resultados presentados.

CUADRO 2. Concordancia de la clasificación de las muertes estudiadas según el registro continuo del Ministerio de Salud Pública y los resultados del presente estudio. Cuba, 2013

\begin{tabular}{|c|c|c|c|c|c|c|}
\hline \multirow{2}{*}{$\begin{array}{l}\text { Clasificación de las } \\
\text { muertes estudiadas según } \\
\text { el registro continuo }\end{array}$} & \multicolumn{6}{|c|}{ Clasificación de las muertes según este estudioa } \\
\hline & $\begin{array}{l}\text { Maternas } \\
\text { directas }\end{array}$ & $\begin{array}{l}\text { Maternas } \\
\text { indirectas }\end{array}$ & $\begin{array}{c}\text { Otras } \\
\text { maternas }^{b}\end{array}$ & $\begin{array}{c}\text { Maternas } \\
\text { tardías }\end{array}$ & No maternas & Total \\
\hline Maternas directas & 27 & 0 & 0 & 0 & 0 & 27 \\
\hline Maternas indirectas & 2 & 20 & 0 & 0 & 0 & 22 \\
\hline Otras maternas ${ }^{\mathrm{b}}$ & 1 & 0 & 8 & 0 & 0 & 9 \\
\hline Maternas tardías & 0 & 0 & 0 & 7 & 0 & 7 \\
\hline No maternas & 0 & 0 & 0 & 1 & 31 & 32 \\
\hline $\begin{array}{l}\text { Total } \\
\text { Concordancia: 95,9\% }\end{array}$ & 30 & 20 & 8 & 8 & 31 & 97 \\
\hline
\end{tabular}

CUADRO 3. Muertes maternas directas, según el método de reporte y las causas de muerte. Cuba, 2013

\begin{tabular}{|c|c|c|c|c|}
\hline \multirow{2}{*}{ Causas de muerte (código)a } & \multicolumn{2}{|c|}{$\begin{array}{c}\text { Registro continuo del } \\
\text { Ministerio de Salud Pública }\end{array}$} & \multicolumn{2}{|c|}{ Este estudio } \\
\hline & No. & $\%$ & No. & $\%$ \\
\hline Complicaciones relacionadas con el puerperio (085-092) & 8 & 29,7 & 9 & 30,0 \\
\hline Embarazo ectópico (000) & 5 & 18,5 & 5 & 16,7 \\
\hline Trastornos hipertensivos (011-016) & 4 & 14,8 & 4 & 13,3 \\
\hline Complicaciones del trabajo de parto y del parto (060-075) & 3 & 11,1 & 4 & 13,3 \\
\hline Aborto, excluye embarazo ectópico (001-008) & 2 & 7,4 & 3 & 10,0 \\
\hline Otras & 5 & 18,5 & 5 & 16,7 \\
\hline Total & 27 & 100,0 & 30 & 100,0 \\
\hline
\end{tabular}

a Codificadas según la Clasificación Estadística Internacional de Enfermedades y Problemas Relacionados con la Salud en su 10. revisión (CIE-10) (28).

Fuente: Elaboración de los autores a partir de los resultados presentados. categorías listadas: complicaciones relacionadas con el puerperio, complicaciones del trabajo de parto y el parto, y el aborto (excluido el embarazo ectópico) (cuadro 3).

En el cuadro 4 se presenta la mortalidad materna indirecta, según la causa básica de muerte notificada por el registro continuo y por este estudio. Se intercambiaron algunos lugares en este indicador y el número de fallecidas varió en solo una unidad en seis de las ocho categorías listadas.

\section{DISCUSIÓN}

Los resultados de esta investigación muestran el alto grado de integridad de las estadísticas cubanas de mortalidad materna, al adicionar solo una defunción a los datos del registro continuo. En el 2012, la Secretaría de Salud de México presentó un estudio sobre muerte materna en el que al confrontar los datos con otras fuentes (formales y no formales) se halló que anualmente incorporan entre $10 \%$ y $30 \%$ de defunciones maternas no clasificadas como tales inicialmente (29).

Se conoce que pueden existir diferencias entre las estimaciones de la mortalidad materna que realiza el MMEIG con las notificadas por los países. En el caso de Cuba, la estimación del MMEIG para 
CUADRO 4. Muertes maternas indirectas según el tipo de metodología y las cinco primeras causas de muerte. Cuba, 2013

\begin{tabular}{|c|c|c|c|c|}
\hline \multirow{2}{*}{ Causas de muerte (código)a } & \multicolumn{2}{|c|}{$\begin{array}{l}\text { Registros continuos del } \\
\text { Ministerio de Salud Pública }\end{array}$} & \multicolumn{2}{|c|}{ Este estudio } \\
\hline & No. & $\%$ & No. & $\%$ \\
\hline Enfermedades del sistema circulatorio (099.4) & 6 & 27,3 & 5 & 25,0 \\
\hline Enfermedades del sistema respiratorio (099.5) & 5 & 22,8 & 6 & 30,0 \\
\hline $\begin{array}{l}\text { Hipertensión preexistente que complica el } \\
\text { embarazo, el parto y el puerperio (010) }\end{array}$ & 4 & 18,2 & 3 & 15,0 \\
\hline $\begin{array}{l}\text { Otras enfermedades maternas clasificables en otra } \\
\text { parte, pero que complican el embarazo, el parto y } \\
\text { el puerperio (099.8) }\end{array}$ & 3 & 13,7 & 4 & 20,0 \\
\hline Enfermedades infecciosas y parasitarias (098) & 1 & 4,5 & 0 & 0 \\
\hline $\begin{array}{l}\text { Trastornos mentales y enfermedades del sistema } \\
\text { nervioso que complican el embarazo, el parto y el } \\
\text { puerperio (099.3) }\end{array}$ & 1 & 4,5 & 1 & 5,0 \\
\hline $\begin{array}{l}\text { Enfermedades del sistema digestivo que } \\
\text { complican el embarazo, el parto y el puerperio } \\
\text { (099.6) }\end{array}$ & 1 & 4,5 & 0 & 0 \\
\hline $\begin{array}{l}\text { Otras enfermedades de la sangre y de los órganos } \\
\text { hematopoyéticos (099.1) }\end{array}$ & 1 & 4,5 & 1 & 5,0 \\
\hline Total & 22 & 100,0 & 20 & 100,0 \\
\hline
\end{tabular}

a Codificadas según la Clasificación Estadística Internacional de Enfermedades y Problemas Relacionados con la Salud en su 10. ${ }^{a}$ Revisión (CIE-10) (28).

Fuente: Elaboración de los autores a partir de los resultados presentados.

el 2013 fue de 80,0 defunciones maternas por 100000 nacidos vivos (13) incluidas las muertes maternas tardías, en contraste con la cifra oficial publicada por Cuba de 38,9 (30), en la que no se incluyen las muertes maternas tardías tal como se reglamenta en la CIE-10 (11).

Al incluir a las dos fallecidas identificadas en el estudio - una como causa materna directa y la otra como causa materna tardía-, la razón de mortalidad materna en Cuba se eleva a 46,1 por 100000 nacidos vivos, lo que aún refleja una sobreestimación de la tasa calculada por el MMEIG de 1,7 veces.

En otros países de la Región, como Brasil, se calculan factores de corrección bianualmente, y en el período 1991-2005 se observaron variaciones que oscilaban entre 1,6 y 1,8 (31). En Argentina se determinó que las muertes maternas subregistradas ocurridas en el año 2008 en los establecimientos de salud de la Ciudad Autónoma de Buenos Aires duplicaron el número de las muertes maternas registradas (32).

Otros estudios indican que hay subregistros en la mortalidad materna debido a la mala calidad de los registros y, en algunos casos, para ocultar las muertes relacionadas con el aborto, ya que está penalizado en el país (12). En Cuba, la interrupción del embarazo es legal y voluntaria, y se realiza de manera segura en hospitales (24).
En el presente estudio, en $20(0,7 \%)$ de las 2731 fallecidas en edad fértil no se pudo comprobar mediante ninguna de las fuentes establecidas en la metodología si tuvieron un embarazo el año previo a su defunción. Estudios realizados en México en el 2011 y el 2012 obtuvieron como resultado $4,7 \%$ (120 de 2523 ) y $2,9 \%$ (113 de 4645$)$, respectivamente $(28,33)$.

$\mathrm{Si}$ a los 20 casos en los que no se pudo comprobar un embarazo previo se les aplicara la lista de causas propuesta por la investigación mexicana realizada según la metodología de RAMOS modificada (29), solo habrían quedado cuatro fallecidas $(0,2 \%)$ : dos por enfermedades hipertensivas, una por infarto agudo de miocardio $y$ otra por insuficiencia cardíaca.

En cuanto a la calidad de la clasificación, se comprobó una variación de tres casos en las muertes directas y una en las tardías - lo que es frecuente en este tipo de estudios-, para una concordancia de $95,9 \%$.

Otras investigaciones han mostrado que cuando se cambia de una clasificación a otra puede variar la codificación (34). En la presente investigación se hallaron cambios en los códigos, que pueden provocar alteraciones en el valor del indicador. También se debe tener en cuenta el cambio en el código de la causa básica de defunción, aun cuando no varíe la clasificación, pues se encontraron casos así tanto en las muertes maternas directas como en las indirectas. En opinión de los autores, en esto pudiera estar influyendo el llenado incorrecto de los certificados médicos de defunción, lo que llevaría a un incorrecto análisis final de la enfermedad que causó la muerte.

En otros estudios se han determinado diversas dificultades que llevan al subregistro de la mortalidad, entre las que se encuentran el déficit en la captación y el registro de la información en el informe estadístico de defunción y en las historias clínicas; la baja cobertura de los sistemas estadísticos; y no valorar la calidad de la información como dimensión de la calidad de la atención, entre otras (31).

En Cuba, se vela por la calidad de la información desde diferentes perspectivas: la información sobre muertes maternas, para efectos estadísticos, está reglamentada en documentos normativos regulatorios de obligatorio cumplimiento; se realizan visitas de control y asesorías integrales y especializadas, así como reuniones y conferencias presenciales y virtuales de las unidades subordinadas con los niveles administrativos superiores y organismos internacionales; se organizan talleres, listas de discusión, y cursos de formación y capacitación tanto nacionales como internacionales; se ejecutan investigaciones; se revisa y actualiza el Sistema de Información Estadístico Complementario cada 5 años; y se llevan a cabo procesos de retroalimentación, como la conciliación entre la estructura de la Oficina Nacional de Estadística e Información, responsable de la información gubernamental, y la estructura estadística de salud del MINSAP (26).

Desde el punto de vista epidemiológico y asistencial, en el Sistema Nacional de Vigilancia en Salud se establece la atención de las mujeres embarazadas y puérperas como un componente fundamental. Además, se han institucionalizado los comités de mortalidad materna en todo el país - que entre otras funciones garantizan la asesoría al personal médico desde el pregrado- y se controla el cumplimiento de lo normado y el llenado correcto del certificado médico de defunción (35).

$\mathrm{Al}$ analizar estos resultados se deben tener en cuenta algunas limitaciones del estudio, como el sesgo de memoria en que se pudo incurrir al aplicar el formulario 3 (autopsia verbal) a familiares y convivientes en 239 casos; sin embargo, esta cifra representó solamente $8,8 \%$ de 
los casos estudiados, por lo que su efecto no es de consideración. La información recogida en el resto de los formularios provino de los documentos oficiales que se emplean en los servicios asistenciales ya descritos.

\section{CONCLUSIONES Y RECOMENDACIONES}

La información sobre las muertes maternas recogida en el registro continuo de mortalidad de la Dirección de
Registros Médicos y Estadísticas de Salud del MINSAP tiene un alto nivel de integridad. La calidad de la clasificación de las muertes maternas en ese registro es elevada, al verificarse muy pocos cambios en la clasificación de las causas de muerte.

A partir de los resultados de este estudio, de alcance nacional, se recomienda publicar la razón de mortalidad materna que emite el país, según los datos de su sistema de información, en lugar de emplear una razón de mortalidad

\section{REFERENCIAS}

1. Mac Dorman MF, Declercq E, Cabral H, Morton C. Recent increases in the U.S. maternal mortality rate: disentangling trends from measurement issues. Obstet Gynecol. 2016;128(3):447-55. Disponible en: http:// europepmc.org/articles / pmc5001799 Acceso el 17 de septiembre del 2017.

2. Say L, Chou D, Gemmill A, Tunçalp Ö, Moller AB, Daniels J, et al. Global causes of maternal death: a WHO systematic analysis. Lancet Glob Health. 2014;2(6):e323-33. Disponible en: http://www.sciencedirect. com/science/article/pii/S2214109X1470 227X\# Acceso el 17 de septiembre del 2017.

3. Santos Rebaza LK, Luna Victoria Mori FM, González Reyes M. Mortalidad materna: factores determinantes modificables mediante políticas públicas en la región La Libertad. UCV Scientia (Caracas). 2010;2(1): 22-33. Disponible en: http://revistas.ucv. edu.pe/index.php/UCV-SCIENTIA/article/view/476/350 Acceso el 17 de septiembre del 2017.

4. Del Carpio Ancaya L. Situation of maternal mortality in Peru, 2000-2012. Rev Peru Med Exp Salud Publica. 2013;30(3):461-4. Disponible en http:/ /www.scielo.org.pe/ scielo.php?script=sci_arttext\&pid=S1726$46342013000300015 \& \operatorname{lng}=$ es\&nrm =iso Acceso el 22 de septiembre del 2017.

5. Maisam Najafizada SM, Bourgeault IL, Labonté R. Social determinants of maternal health in Afghanistan: a review. Centr Asian J Glob Health. 2017;6(1). Disponible en: https://cajgh.pitt.edu/ojs/index.php/ cajgh/article/view/240 Acceso el 17 de septiembre del 2017.

6. Cordero-Rizo MZ, González-Pérez GJ. Factores socio económicos y de servicios de salud asociados con la mortalidad materna: una revisión. Rev Cienc Biomedicas 2011;2(1):71-85 Disponible en: https:// www.researchgate.net/publication / 260776797_Experiencias_y_sentimientos_ vividos_durante_una_gestacion_de_alto_ riesgo_un_estudio_documental_2005-2011 Acceso el 27 de septiembre del 2017.

7. Ronsmans C, Graham WJ. Maternal mortality: who, when, where, and why. Lancet. 2006;368(9542):1189-200. Disponible en: http:/ / www.thelancet.com/pdfs/journals/lancet/PIIS014067360669380X.pdf Acceso el 28 de septiembre del 2017.
8. Small MJ, Allen TK, Haywood L, Brown MD. Global disparities in maternal morbidity and mortality. Semin Perinatol. 2017;1(5):318-22. Disponible en: http:// www.sciencedirect.com/science/article/ pii/S0146000517300514 Acceso el 17 de septiembre del 2017.

9. Freyermuth EG. Determinantes sociales en la mortalidad materna en México. Rev CONAMED. 2016;21(1). Disponible en: http:/ / www.dgdi-conamed.salud.gob. $\mathrm{mx} /$ ojs-conamed/index.php/revconamed/article/view/400/709 Acceso el 17 de septiembre del 2017

10. Hogan MC, Foreman KJ, Naghavi M, Yahn S, Wang M, Makela SM, et al. Maternal mortality for 181 countries, 1980-2008: a systematic analysis of progress towards Millennium Development Goal 5. Lancet. 2010;375(9726):1609-23. Disponible en: http:/ / www.sciencedirect.com/science/ article/pii/S0140673610605181 Acceso el 2 de octubre del 2017

11. Ruiz Salguero MT, Márquez L, Miller T. La mortalidad materna: ¿por qué difieren las mediciones externas de las cifras de los países? Santiago: Comisión Económica para América Latina y el Caribe; 2011. (Serie Población y Desarrollo N. ${ }^{\circ} 113$ ). Disponible en: http://200.9.3.98/bitstream/handle/11362/39414/S1500951_ es.pdf? sequence $=1 \&$ isAllowed $=y$ Acceso el 9 de octubre del 2017.

12. Jentoft S, Vibeke Oestreich N, Roll-Hansen D. Adjusting maternal mortality data for international comparisons. The case of vital registration systems. Statistics Norway. 2014;(773). Disponible en: http://www. ssb.no/en/forskning/discussion-papers/ _attachment $/ 168855$ ?_ts=144de574468 Acceso el 14 de octubre del 2017.

13. World Health Organization, United Nations International Children's Fund, United Nations Population Fund, The World Bank, United Nations. Trends in maternal mortality: 1990 to 2013. Estimates by WHO, UNICEF, UNFPA, The World Bank and the United Nations Population Division. Geneva: WHO, UNICEF, UNFPA, WB, UN; 2013. Disponible en: http: / / apps.who.int/iris / bitstre am/10665/112682/2/9789241507226_eng. pdf Acceso el 17 de septiembre del 2017. materna afectada por la aplicación de un factor de ajuste proveniente de otros estudios o supuestos metodológicos.

\section{Conflicto de intereses. Ninguno.}

Declaración. Las opiniones expresadas en este manuscrito son responsabilidad de los autores y no reflejan necesariamente los criterios ni la política de la Revista Panamericana de Salud Pública / Pan American Journal of Public Health o de la Organización Panamericana de la Salud.
14. Creanga A, Callaghan W. Recent increases in the U.S. maternal mortality rate: disentangling trends from measurement issues. Obst Gynecol. 2017;129(1):206-7. Disponible en: http://journals.lww.com/greenjournal / Citation / 2017/01000/Recent Increases_in_the_U_S__Maternal_ Mortality.36.aspx Acceso el 23 de septiembre del 2017.

15. Márquez L, Plana A, Villarroel MC. Mortalidad materna en pueblos indígenas y fuentes de datos: alcances y desafíos para su medición en países de América Latina. Santiago: Comisión Económica para América Latina y el Caribe; 2015. (Serie Población y Desarrollo N. ${ }^{\circ} 118$ ). Disponible en: http:/ / repository.eclac.org/bitstream/ handle/11362/42029/S1700558_es.pdf? sequence $=1 \&$ isAllowed $=y$ Acceso el 23 de septiembre del 2017.

16. Organización Mundial de la Salud. Mortalidad materna en 2005. Estimaciones elaboradas por la OMS, el UNICEF, el UNFPA y el Banco Mundial. Ginebra: OMS, UNICEF, UNFPA, BM; 2008. Disponible en: http://apps.who.int/iris/ bitstream/10665/43849/1/9789243 596211_spa.pdf Acceso el 23 de septiembre del 2017.

17. Cobos MI, Miller T, Ruiz Saguero M. Hacia la armonización de las estimaciones de mortalidad materna en América Latina. Hallazgos de un estudio piloto en ocho países. Santiago: Comisión Económica para América Latina y el Caribe; 2013. (Serie Población y Desarrollo N. ${ }^{\circ}$ 108). Disponible en: http://repositorio.cepal. org/bitstream/handle/11362/7143/1/ LCL3735_es.pdf Acceso el 23 de septiembre del 2017.

18. Ruiz Salguero MT, Miller T, Márquez L, Villarroel MC. Hacia la armonización de las estimaciones de mortalidad materna en América Latina: actualización y ampliación a los 20 países de la región. Santiago: Comisión Económica para América Latina y el Caribe; 2015. (Serie Población y Desarrollo N.' 111). Disponible en: http:// repositorio.cepal.org/bitstream/handle/11362/39297/1/S1500944_es.pdf Acceso el 23 de septiembre del 2017.

19. Torres LM, Rhenals AL, Jiménez A, Ramírez-Villalobos D, Urióstegui R, Piña 
$\mathrm{M}$, et al. Búsqueda intencionada y reclasificación de muertes maternas en México: el efecto en la distribución de las causas. Salud Publica Mex. 2014;56:333-47.

20. Herrera Alcázar VR, Presno Labrador MC, Torres Esperón JM, Fernández Díaz IE, Martínez Delgado DA, Machado Lubián MC. Consideraciones generales sobre la evolución de la medicina familiar y la atención primaria de salud en Cuba y otros países. Rev Cubana Med Gen Integr. 2014; 30(3):364-74. Disponible en: http://scielo. sld.cu/scielo.php?script=sci_arttext\& pid=S0864-21252014000300010\&lng=es Acceso el 23 de septiembre del 2017.

21. Verdecia Jiménez AI. Quality of death medical certification. CCM (Holguín, Cuba). 2013;17(3):372-4. Disponible en: http:// scielo.sld.cu/scielo.php?script=sci_arttext\& pid=S1560-43812013000300018\&lng =es Acceso el 30 de septiembre del 2017.

22. Cuba, Ministerio de Salud Pública, Sistema de Información Estadística Nacional. Modelo 8111, certificado médico de defunción (para fallecidos de 28 días y más). La Habana: MINSAP; 2012. Disponible en: http:/ / files.sld.cu/dne/files/2012/03/cmd_28_mas.pdf Acceso el 30 de septiembre del 2017.

23. Zacca Peña E, Gran Álvarez MA, Martínez Morales MA, Fernández Viera MR. Calidad de las estadísticas de mortalidad en Cuba según cuantificación de causas de muerte imprecisas. Rev Cubana Salud Publica. 2010;36(2):102-8. Disponible en: http:/ / scielo.sld.cu/scielo.php?script= sci_arttext\&pid=S0864-34662010000200002 Acceso el 30 de septiembre del 2017.

24. Gran Álvarez MA, Zacca Peña E, Martínez Morales MA, Fernández Viera MR. Calidad de las estadísticas de mortalidad en provincias cubanas según cuantificación de causas de muerte imprecisas. Rev Cubana Salud Publica. 2010;36(2)109-15. Disponible en: http://scielo.sld.cu/scielo. php?script=sci_arttext\&pid=S0864-34662
010000200003 Acceso el 23 de septiembre del 2017.

25. Gran Álvarez M, Torres Vidal R, López Nistal L, Pérez Leyva M. Fecundidad, anticoncepción, aborto y mortalidad materna en Cuba. Rev Cubana Salud Publica. 2013;39(supl 1):822-35. Disponible en: http://scielo.sld.cu/scielo.php?script=sci arttext\&pid $=$ S0864-34662013000500003 Acceso el 23 de septiembre del 2017.

26. Gran Álvarez M, Ramil J, Peraza Peraza M, Pérez Leyva M, Martínez Morales M, Pérez ER. Sistema de información estadística de salud cubano. La Habana: MINSAP; 2002.

27. México, Instituto Nacional de Estadística y Geografía. Método RAMOS: Reproductive Age Mortality Survey [presentación en PowerPoint]. México, DF: INEGI; sf. Disponible en: http://iieg.gob.mx/contenido / Poblacion Vivienda / Salud / ResumenMetodoRamos.pdf Acceso el 30 de septiembre del 2017.

28. Organización Panamericana de la Salud. CIE-10. Clasificación Estadística Internacional de Enfermedades y Problemas Relacionados con la Salud. 10. ${ }^{\mathrm{a}}$ revisión. Washington, DC: OPS; 2008. (Publicación científica No. 54).

29. México, Secretaría de Salud. Manual de procedimientos para la búsqueda intencionada y reclasificación de muertes maternas. Informe 2011. México, DF: Secretaría de Salud; 2012. Disponible en: http:/ / www.dgis.salud.gob.mx/descargas / pdf / BIRMM_Informe2011.pdf Acceso el 30 de septiembre del 2017.

30. Cuba, Ministerio de Salud Pública. Anuario Estadístico de Salud. 2015. La Habana: MINSAP; 2016. Disponible en: http:/ / files.sld.cu/dne/files / 2016/04/ Anuario_2015_electronico-1.pdf Acceso el 30 de septiembre del 2017.

31. Muniz Néquern Soares N, Minorelli de Azevedo EM, Watanabe TL. Subnotificação da mortalidade materna no estado do Paraná, Brasil: 1991-2005. Cad Saude
Publica. 2008;24(10):2418-26. Disponible en: http:/ / www.scielo.br/scielo.php?script= sci_arttext\&pid=S0102-311X2008001000022 Acceso el 30 de septiembre del 2017.

32. Argentina, Dirección General de Estadística y Censos. Omisión de registro de causas de muertes maternas en establecimientos de salud de la CABA. Año 2008. Buenos Aires: Dirección General de Estadística y Censos; 2012. Disponible en: https:/ / www.estadisticaciudad.gob.ar/ eyc/wp-content/uploads/2015/04/mortalidad_materna_2012_octubre.pdf Acceso el 30 de septiembre del 2017.

33. México, Secretaría de Salud. Búsqueda Intencionada y Reclasificación de Muertes Maternas -BIRMM- en México. Informe 2012-2013. México, DF: Secretaría de Salud; 2016. Disponible en: http://www. dgis.salud.gob.mx/descargas/pdf / Muertes_Maternas_11_Enero_2016.pdf Acceso el 30 de septiembre del 2017.

34. Joseph KS, Lisonkova S, Muraca G, Razaz N, Sabr Y, Mehrabadi A, et al. Factors underlying the temporal increase in maternal mortality in the United States. Obstet Gynecol. 2017;129(1):91-100. Disponible en: http://journals.lww.com/greenjournal/Abstract/2017/01000/Factors Underlying_the_Temporal_Increase_ in.12.aspx Acceso el 2 de octubre del 2017.

35. Cuba, Ministerio de Salud Pública. Reglamento general de hospitales. La Habana: MINSAP; 2007. Disponible en: http:// files.sld.cu/minsapdocumentos/ files / 2009 / 08 / reglamento-generalde-hospitales.pdf Acceso el 2 de octubre del 2017.

Manuscrito recibido el 8 de julio del 2017. Aceptado para publicación, tras revisión, el 11 de enero del 2018. 
ABSTRACT Objective. Evaluate the degree of integrity of the Cuban statistical registry of maternal deaths and the quality of the classification of the causes of death included in that registry.

\section{Quality of maternal mortality statistics in Cuba, 2013}

Keywords Maternal mortality; epidemiological surveillance; quality control; death certificates;

Cuba. in 2013 according to the continuous mortality registry of the Directorate of Medical Records and Health Statistics of the Ministry of Public Health of Cuba (MINSAP), regardless of the main cause of death informed. Four research groups (national, expert, provincial and health units), each with defined functions, applied four forms to establish if the women had been pregnant in the year prior to death, to reassess whether the cases corresponded to a maternal death and, in that case, to review the classification.

Results. Deaths of 2731 women of reproductive age notified in Cuba in 2013 were assessed. Of them, the cause of death of 2711 (99.3\%) was conclusive and, of these, $97(3.6 \%)$ had had a pregnancy in the year prior to death. We found 50 maternal deaths (one more than in the continuous registry) for a $2 \%$ error and an adjustment factor of 1.02. Of the 97 deaths studied, only 4 cases were reclassified: 2 maternal deaths and 1 death related to pregnancy, delivery and puerperium, according to the continuous registry, which were reclassified as direct deaths; and 1 death considered non-maternal by the continuous registry that was reclassified as late maternal death, for a $95.9 \%$ concordance.

Conclusions. The information on maternal deaths included in the MINSAP's continuous mortality registry has a high level of integrity. The quality of the classification of maternal deaths in this registry is high; reclassification of causes of death is uncommon.

Methods. We analyzed the information of all Cuban women who died in fertile age

RESUMO

\section{Qualidade das estatísticas de mortalidade materna em Cuba, 2013}

Palavras-chave
Objetivos. Avaliar o grau de integridade do registro estatístico cubano de óbitos maternos e a qualidade da classificação das causas de óbito incluídas nesse registro.

Métodos. Analisamos a informação de todas as mulheres cubanas que morreram em idade fértil em 2013 de acordo com o histórico de mortalidade contínua da Diretoria de Registros Médicos e Estatísticas de Saúde do Ministério da Saúde Pública de Cuba (MINSAP), independentemente da causa básica da morte consignada. Quatro grupos de pesquisa (nacional, especialistas, provincial e unidades de saúde), com funções definidas, aplicaram quatro formas para determinar se houve gravidez no ano anterior à morte, reavaliam se os casos corresponderam a uma morte materna e, em nesse caso, rever a classificação.

Resultados. Foram investigadas as 2731 mulheres falecidas de idade reprodutiva notificadas em Cuba em 2013, das quais as causas de morte de 2711 (99,3\%) foram conclusivas e, destas, 97 (3,6\%) tiveram gravidez no ano anterior à morte. Encontramos 50 mortes maternas (uma mais do que no registro contínuo) por erro de $2 \%$ e um fator de ajuste de 1,02. Das 97 mortes estudadas, apenas 4 casos foram reclassificados: 2 mortes maternas e 1 morte relacionada à gravidez, parto e puerpério, de acordo com o registro contínuo, que foram reclassificadas como mortes diretas, e 1 morte considerada não materna pelo registro contínuo que foi reclassificada como morte materna tardia, para uma concordância de $95,9 \%$.

Conclusões. A informação sobre mortes maternas incluída no registro de mortalidade contínua do MINSAP tem alto nível de integridade. A qualidade da classificação das mortes maternas neste registro é alta; a reclassificação das causas de morte é pouco frequente.

Mortalidade materna; vigilância epidemiológica; controle de qualidade; atestado de óbito; Cuba. 